\title{
ErbB4 in Spinal PV Interneurons Regulates Mechanical Allodynia in Neuropathic Pain via Modulation of Glycinergic Inhibitory Tone
}

\author{
Yingying Yu \\ Guohua Wei \\ Qi Zhou \\ Huanhuan Sha \\ Department of Anesthesiology and \\ Perioperative Medicine, The First \\ Affiliated Hospital of Nanjing Medical \\ University, Nanjing, Jiangsu, 210029 , \\ People's Republic of China
}

Background: Mechanical allodynia is the most common and challenging symptom associated with neuropathic pain; however, the underlying mechanisms are still unclear. The aim of this study was to investigate whether ErbB4, a receptor for neuregulin-1 (NRG1), participates in the modulation of mechanical allodynia.

Methods: Radiant heat and von Frey filaments were applied to assess nociceptive behaviors. Real-time quantitative polymerase chain reaction, Western blotting, immunofluorescence, and small interfering RNA were used to identify the likely mechanisms.

Results: ErbB4 was rapidly and persistently activated in spinal parvalbumin (PV) interneurons after chronic constriction injury (CCI) in mice. Knockdown of ErbB4 in the spinal cord prevented and reversed CCI-induced mechanical allodynia, and activation of ErbB4 by spinal application of NRG1 induced mechanical allodynia in naïve mice. Furthermore, we found that activation of ErbB4 decreased the glycine concentration in the spinal cord, contributing to modulation of mechanical allodynia.

Conclusion: ErbB4 in spinal PV interneurons gates mechanical allodynia in neuropathic pain via regulation of glycinergic inhibitory tone, suggesting that a possible ErbB4-mediated process participates in the development of neuropathic pain.

Keywords: Erb4, mechanical allodynia, parvalbumin interneuron, glycine

\section{Introduction}

Neuropathic pain is chronic pain due to direct central or peripheral nerve damage or disease. Neuropathic pain sufferers often experience depression, anxiety and sleep disturbances accompanied by pain, leading to a significantly reduced quality of life. Mechanical allodynia, a type of evoked pain that is elicited by nonpainful mechanical stimulation (eg, light touch), is the most common and challenging symptom of neuropathic pain. The gate control theory predicts that inhibitory interneurons in the substantia gelatinosa (lamina II) of the spinal cord act as "gate control" units for the interaction between innocuous and nociceptive signals. ${ }^{1}$ Under normal conditions, the innocuous and nociceptive pathways are kept separated in the spinal cord by tonic inhibition derived from inhibitory interneurons. However, in the setting of nerve injury, inhibitory control is reduced, thereby enabling innocuous inputs to engage and activate nociceptive pathways. Mechanical allodynia in neuropathic pain may thus result from dysfunction of spinal inhibitory interneurons, leading to miscoding of tactile information by cells that normally only respond to painful stimuli. $^{2-4}$
Correspondence: Huanhuan Sha Department of Anesthesiology and Perioperative Medicine, The First Affiliated Hospital of Nanjing Medical University, Nanjing, Jiangsu, 210029

People's Republic of China

Tel/Fax +86-25-837I-45II

Email shasha_1985@I26.com 
Recent studies have indicated that parvalbumin (PV) interneurons are present in the adult brain and play a critical role in a variety of neurological and psychiatric disorders, including Alzheimer's disease, schizophrenia, and epilepsy. ${ }^{5-8}$ Interestingly, PV interneurons are also present in the adult spinal cord. Within the superficial dorsal horn, PV-immunoreactive neurons are concentrated in laminae II and III. ${ }^{9,10}$ Most PV-immunoreactive neurons in the dorsal horn are GABA-immunoreactive neurons, and all of them are glycine-immunoreactive neurons. ${ }^{11}$ Recently, by using genetic and functional approaches, Petitjean et al demonstrated that PV interneurons in the spinal cord are modality-specific filters that gate mechanical but not thermal inputs to the dorsal horn. ${ }^{12}$ Ablation or silencing of PV interneurons induces mechanical allodynia in naïve mice via disinhibition of PKC $\gamma$ excitatory interneurons. Conversely, increasing PV interneuron activity alleviates the mechanical allodynia that develops following nerve injury. These findings suggest that PV interneurons in the dorsal horn play an important role in gating mechanical allodynia after peripheral nerve injury. However, the molecular mechanisms that prevent this process are still unidentified.

ErbB4, a receptor for neuregulin-1 (NRG1), is widely expressed throughout the central nervous system and has been reported to be involved in a variety of neurophysiological and neuropathological processes, such as neuronal development, synaptic plasticity, and CNS diseases. ${ }^{13,14}$ NRG1 causes dimerization and activation of ErbB4 and subsequent phosphorylation of its intracellular domains (ICDs) and creates docking sites for adaptor proteins including Grb2 and Shc for Erk activation, for $\mathrm{p} 85$ for PI3K activation, and for the Src kinases Pyk2 and Cdk5, and PLCg. ${ }^{14}$ Recent evidence indicates that ErbB4 mRNA is enriched in regions where interneurons are clustered. ${ }^{15}$ Furthermore, ErbB4 expression is largely confined to specific classes of interneurons, particularly parvalbumin-expressing neurons, in the adult brain. ${ }^{16-19}$ Previous studies have reported that ErbB4 in PV interneurons is critical for NRG1-mediated regulation of pyramidal neuronal activity and long-term potentiation (LTP). ${ }^{18,20}$ In the present study, by using a sciatic nerve chronic constriction injury (CCI) model, we found that ErbB4 was selectively expressed in spinal PV interneurons and is a potential drug target for the prevention and alleviation of mechanical hypersensitivity.

\section{Methods and Materials}

\section{Animals and Surgeries}

Adult male C57BL/6J mice were obtained from and housed in the Center of Experimental Animals of Zhejiang Chinese Medical University. All animal procedures were reviewed and approved by the Animal Care and Use Committee of Nanjing Medical University and Zhejiang Chinese Medical University and were performed in accordance with the guidelines of the International Association for the Study of Pain. Left sciatic nerve chronic constriction injury (CCI), ${ }^{21}$ left L5 spinal nerve ligation (SNL), ${ }^{22}$ and spared nerve injury (SNI, left panel $)^{23}$ models of neuropathic pain were generated as previously described. In sham-operated mice, the nerve was exposed but not ligation or transected.

\section{Drugs and Administration}

ErbB4 siRNA (5'-GCCCTCAACCAGTTTCGTT-3') and a scrambled negative control siRNA (5'GCCCAACTGACCTTTCGTT-3') were designed and synthesized by GenePharma Company (Shanghai). The $\gamma$ aminobutyric acid (GABA) receptor antagonists bicuculline and saclofen, the glycine receptor antagonist strychnine, the GABA receptor agonists muscimol and baclofen, glycine, protease inhibitor cocktail, and phosphatase inhibitor cocktails 2 and 3 were purchased from Sigma. NRG1, a recombinant polypeptide containing the entire EGF domain of $\beta$-type NRG1, was purchased from Prospec. Antibodies against ErbB4, phospho-ErbB4 (p-ErbB4, Tyr 1056), and parvalbumin were purchased from Santa Cruz Biotechnology. An antibody against GAPDH and a horseradish peroxidase (HRP)-linked goat anti-rabbit secondary antibody were purchased from Cell Signaling Technology (CST). Alexa Fluor 488- and 594-conjugated goat anti-rabbit secondary antibodies were purchased from Molecular Probe. Drugs were delivered into the subarachnoid space by intrathecal injection with a $28 \mathrm{G}$ stainless steel needle between the L5 and L6 vertebrae. ${ }^{24}$ A successful spinal puncture was evidenced by a brisk tail flick when the needle entered the subarachnoid space.

\section{Behavioral Test}

The paw withdrawal latency (PWL) in response to a thermal stimulus and paw withdrawal threshold (PWT) in response to a mechanical stimulus were measured as previously described. ${ }^{24}$ To assess the PWL, mice were placed in a plastic box on a glass plate and allowed to 
habituate for 1 hour. The plantar surface of the hind paw was exposed to radiant heat through a transparent glass surface. The baseline values were adjusted to $12-15 \mathrm{~s}$, and an automatic $25 \mathrm{~s}$ cutoff was used to prevent tissue injury. The average of 3 values obtained at 5-min intervals was calculated. To evaluate the PWT, mice were placed in a plastic box on a metal mesh floor. The plantar surface was stimulated with a series of von Frey filaments of increasing strength (starting with $0.31 \mathrm{~g}$ and ending with 4.0 g). The PWT was determined using Dixon's up-anddown method. ${ }^{25}$

\section{Western Blot Analysis}

Protein samples were prepared as previously described. ${ }^{24}$ Protein was separated using SDS-PAGE and transferred onto polyvinylidene difluoride membranes. After blocking with milk, the blots were incubated with primary antibodies against ErbB4, p-ErbB4, and GAPDH overnight at $4^{\circ}$ C. These blots were then extensively washed and further incubated with HRP-conjugated secondary antibody at room temperature for 2 hours. The immune complexes were detected by using chemiluminescence (Pierce). The intensity of each band was determined using ImageJ software.

\section{Immunofluorescence}

Mice were deeply anesthetized (sodium pentobarbital, $60 \mathrm{mg} / \mathrm{kg}$, intraperitoneal injection) and intracardially perfused with 4\% paraformaldehyde (PFA). The L4-5 segments of the spinal cord were quickly removed, postfixed with 4\% PFA, cryoprotected in 30\% sucrose, and cut into $30 \mu \mathrm{m}$ sections. After blocking and permeabilization, the sections were incubated with antibodies against p-ErbB4 and parvalbumin overnight at $4^{\circ} \mathrm{C}$. The following day, the sections were incubated with an Alexa Fluor 488- or 594conjugated secondary antibody at room temperature for 2 hours. PBS was used in place of the primary and secondary antibodies as a blank control. The sections were subsequently washed and mounted for confocal imaging.

\section{Measurement of GABA and Glycine Contents}

The dorsal horn of the L4-5 spinal cord segments was quickly dissected and stored in liquid nitrogen. After weighing, the tissue samples were homogenized in sulfosalicylic acid. The homogenates were centrifuged at 12,000 rpm for $15 \mathrm{~min}$ at $4^{\circ} \mathrm{C}$. The supernatant was collected and stored at $-20^{\circ} \mathrm{C}$. The concentrations of GABA and glycine were measured with a S443D automatic amino acid analyzer.

\section{Statistical Analysis}

The data are expressed as mean \pm SD. $P<0.05$ was considered statistically significant. Differences between two groups were analyzed using Student's $t$-test. Differences between more than two groups were analyzed using oneway ANOVA followed by Tukey's post hoc test. The behavioral data were analyzed by two-way repeatedmeasures ANOVA followed by Tukey's post hoc test.

\section{Results}

\section{$\mathrm{CCl}$ Increases the Activation of ErbB4 in the Spinal Cord}

The CCI model has been widely used in preclinical neuropathic pain research. ${ }^{21}$ In the present study, we found that $\mathrm{CCI}$ induced rapid and persistent mechanical allodynia and thermal hyperalgesia in mice (Figure 1A and B). To investigate whether ErbB4 in the spinal cord is associated with the development of neuropathic pain, we first assessed the time course of spinal p-ErbB4 and ErbB4 expression after $\mathrm{CCI}$ or sham surgery. The results showed that CCI induced a long-lasting increase in p-ErbB4 expression (Figure 1C). No significant difference in ErbB4 expression was detected. For comparison, we also assessed the expression of spinal p-ErbB4 and ErbB4 in two other neuropathic pain models, the sciatic nerve ligation (SNL) and sciatic nerve injury (SNI) models. Consistent with CCI, both SNL and SNI significantly increased the expression of p-ErbB4, but not ErbB4, on day 7 after surgery (Figure 1D). Moreover, by using immunofluorescence staining, we further confirmed a significant increase in p-ErbB4 expression in the spinal cords of CCI mice (Figure 2). Additionally, immunofluorescence staining showed that the CCI-induced increase in the expression of p-ErbB4 occurred exclusively in neurons and mainly in PV interneurons of laminae III of the spinal cord (Figure 2A and B). These results suggest that alterations in p-ErbB4 expression in spinal PV interneurons may be critical for the development of neuropathic pain.

\section{Manipulation of ErbB4 Expression in the Spinal Cord Regulates Mechanical Allodynia}

To determine whether the increase in spinal p-ErbB4 levels contributes to pain behaviors in CCI mice, we used ErbB4 siRNA to silence ErbB4 gene expression and 

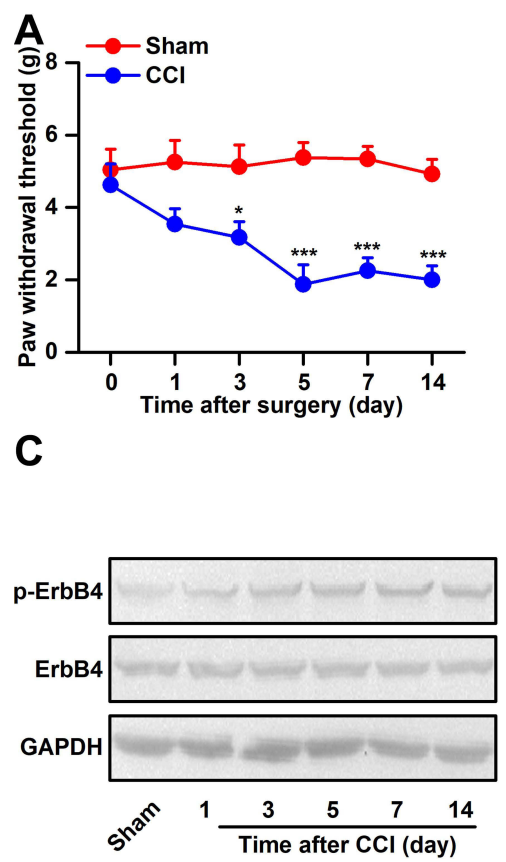
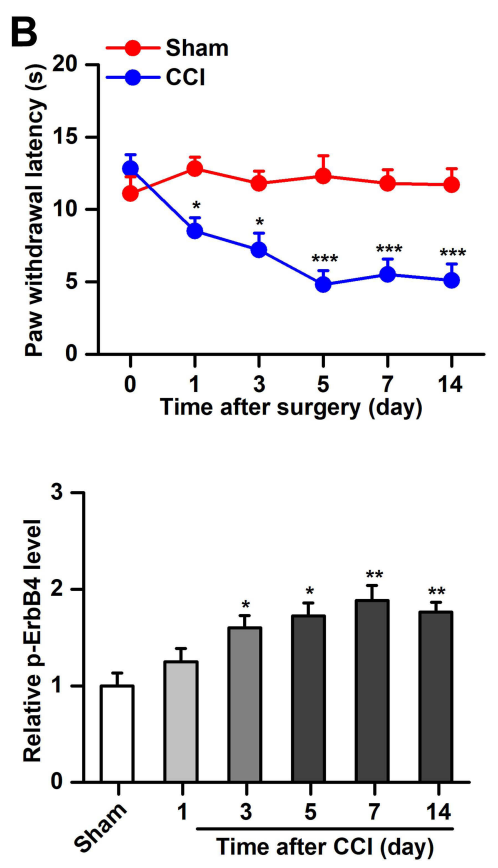
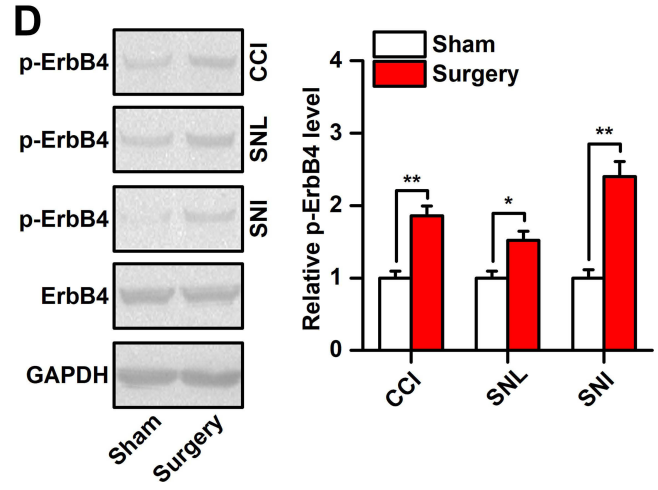

Figure I Alterations in ErbB4 activation after $\mathrm{CCl}$. (A and $\mathbf{B})$, Mechanical allodynia $(\mathbf{A})$ and heat hyperalgesia $(\mathbf{B})$ was observed in the ipsilateral hindpaw after $\mathrm{CCl}$, $\mathrm{n}=\mathbf{8}$. (C), Time course of ErbB4 and phosphor-ErbB4 (p-ErbB4) expression in the spinal cord after chronic constriction injury (CCl) or sham surgery, the representative Western blots were shown in left and the quantitative data was shown in right, $\mathrm{n}=6$. (D), ErbB4 activation in the spinal cord on day 7 after $(\mathrm{CCl}, \mathrm{SNL})$, and $\mathrm{SNI}, \mathrm{n}=6$.

Notes: $* \mathrm{P}<0.05, * * \mathrm{P}<0.01$, $* * * \mathrm{P}<0.001$.

Abbreviations: ErbB4, Erb-B2 receptor tyrosine kinase 4; $\mathrm{CCl}$, chronic constriction injury; SNL, sciatic nerve ligation; SNI, sciatic nerve injury.

then assessed pain behavior at specific points in time. The efficiency of siRNA delivery to the mouse spinal cord by intrathecal injection was validated by Western blotting (Figure 3A). The behavioral results showed that pretreatment with ErbB4 siRNA, but not the scramble control siRNA, from day 0 to day 2 after CCI prevented the induction of mechanical allodynia but not thermal hyperalgesia in mice (Figure 3B and C). Furthermore, posttreatment with ErbB4 siRNA, but not scramble siRNA, from day 7 to day 9 after CCI significantly reversed the already established mechanical allodynia in mice (Figure 3D and E). No significant alterations in thermal hyperalgesia were detected. Since knockdown of ErbB4 in the spinal cord can attenuate mechanical allodynia, can activation of ErbB4 in the spinal cord produce mechanical hypersensitivity? NRG1, a growth and differentiation factor, has been reported to be capable of activating ErbB4, ${ }^{13}$ and its expression is upregulated in the spinal cord after peripheral nerve injury. ${ }^{26}$ Thus, intrathecal injection of NRG1 was used to activate spinal ErbB4 in the present study. The activation of ErbB4 by NRG1 was validated by Western blotting (Figure 3F). Activation of ErbB4 via intrathecal injection of NRG1, but not vehicle control, induced a significant decrease in the mechanical pain threshold in naïve mice (Figure 3G). However, to our surprise, spinal administration of NRG1 also induced a significant reduction in thermal pain latency (Figure 3H). Moreover, NRG1-induced mechanical allodynia, but not thermal hyperalgesia, was dependent on the spinal ErbB4 receptor (Figure 3I and J). Because the interaction between NRG1 and ErbB4 is not specific, we proposed that NRG1-induced thermal hyperalgesia may occur in an ErbB4-independent manner. Together, these findings indicate that activation of ErbB4 in the spinal cord contributes to the modulation of mechanical allodynia in neuropathic pain.

\section{ErbB4 in the Spinal Cord Modulates Mechanical Allodynia by Increasing Glycine Release}

$\mathrm{PV}$ interneurons in the spinal cord express the transmitters GABA and glycine. ${ }^{11}$ ErbB4 in PV interneurons has been reported to modulate the release of inhibitory transmitters. ${ }^{27}$ Considering the causal link between PV interneuron-mediated disinhibition and the development of mechanical allodynia, ${ }^{12}$ we hypothesize that 

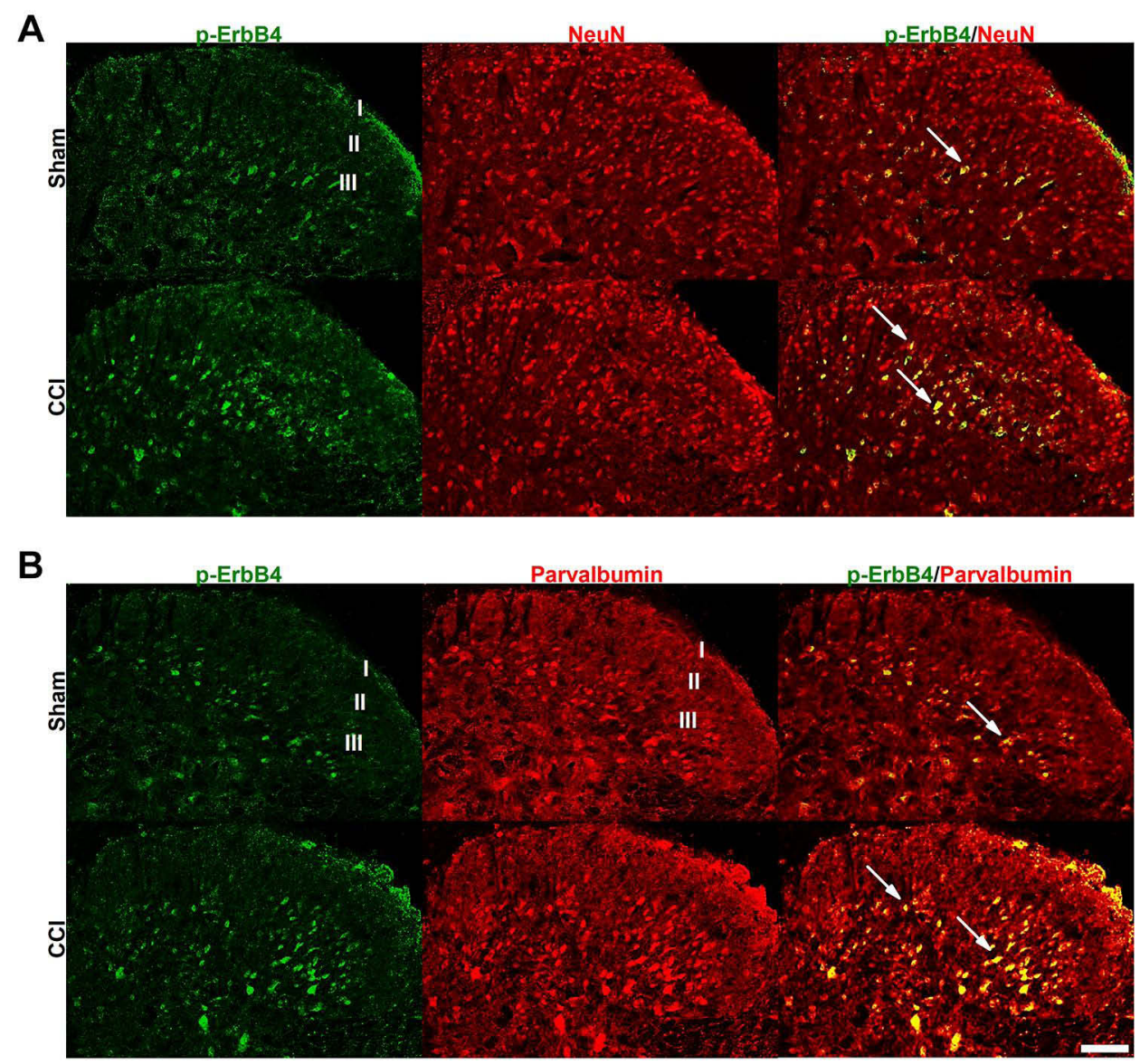

Figure 2 Cellular localization of p-ErbB4 expression in the spinal cord. (A and B), Combined p-ErbB4 and NeuN (a neural marker) (A) or parvalbumin (B) immunofluorescence staining in the spinal cord on day 7 after $\mathrm{CCl}$ or sham surgery. Scale bar, $25 \mu \mathrm{m}$.

Abbreviations: ErbB4, Erb-B2 receptor tyrosine kinase 4; $\mathrm{CCl}$, chronic constriction injury.

dysfunction of inhibitory transmitter release from spinal $\mathrm{PV}$ interneurons is involved in the modulation of mechanical allodynia by ErbB4. To experimentally validate this hypothesis, we first measured the concentrations of GABA and glycine in the spinal cord. The results showed that $\mathrm{CCI}$ induced significant decreases in GABA and glycine contents on day 7 after CCI (Figure 4A and B). However, following knockdown of spinal ErbB4, the CCI-induced decrease in glycine content, but not GABA content, was significantly reversed (Figure 4A and B). Additionally, spinal glycine concentrations, but not GABA concentrations, were decreased by activation of ErbB4 induced by intrathecal injection of NRG1 in naïve mice (Figure 4C and D). To determine the regulatory role of ErbB4 in GABA and glycine release at the behavioral level, we treated mice with antagonists of the GABA or glycine receptor after intrathecal injection of ErbB4 siRNA and then observed the behavioral response. The results revealed that the ErbB4 siRNA-induced increase in the mechanical pain threshold was reversed by intrathecal injection of a glycine receptor antagonist in CCI mice (Figure 4F). No significant alterations were detected after GABA receptor antagonist application (Figure 4E). Consistently, intrathecally injected NRG1-induced mechanical allodynia was inhibited by spinal administration of a glycine receptor agonist, but not a GABA receptor agonist, in naïve mice (Figure $4 \mathrm{G}$ and $\mathrm{H}$ ). It is worth noting that NRG1-induced thermal hyperalgesia was unaffected by the application of either a glycine receptor agonist or a GABA receptor agonist to the spinal cord (Figure $4 \mathrm{I}$ and $\mathrm{J})$. These findings suggest that glycine-mediated disinhibition is required for the modulation of mechanical allodynia related to ErbB4 in the spinal cord.

\section{Discussion}

The present study reveals that ErbB4 in PV interneurons is implicated in the regulation of mechanical allodynia induced by peripheral nerve injury. The major findings 

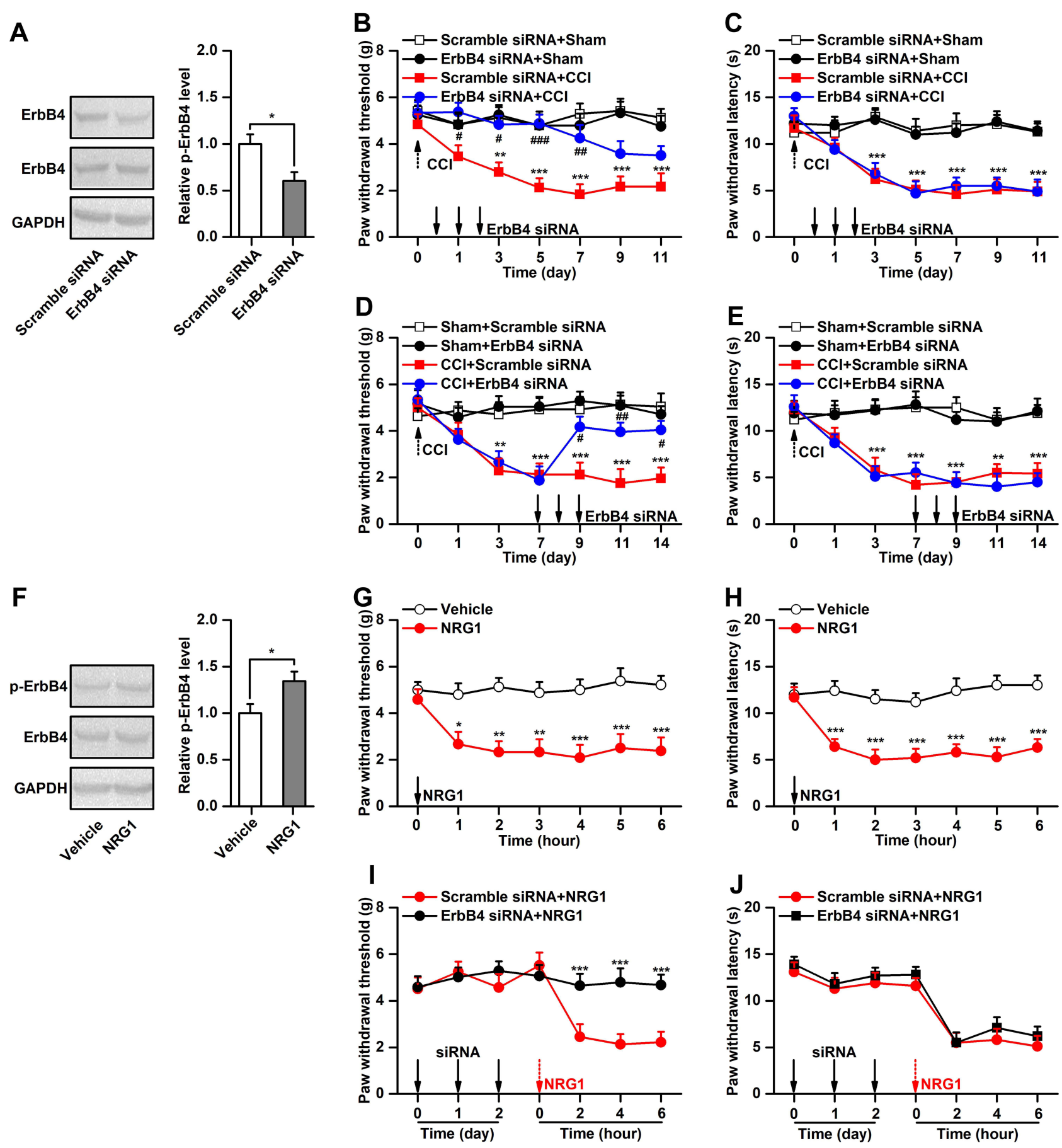

Figure 3 Manipulation of ErbB4 in the spinal cord regulates pain behaviors. (A), The validation of ErbB4 siRNA transfection efficiency in vivo, $n=4$. (B and C), Effect of ErbB4 siRNA $(10 \mu \mathrm{g} / 5 \mu \mathrm{L})$ on the development of $\mathrm{CCl}$-induced mechanical allodynia (B) and thermal hyperalgesia $(\mathbf{C})$, $\mathrm{n}=8$. (D and $\mathbf{E})$, Effect of ErbB4 siRNA (I0 $\mu \mathrm{g} / 5 \mu \mathrm{L})$ on the maintenance of $\mathrm{CCl}$-induced mechanical allodynia (D) and thermal hyperalgesia $(\mathbf{E}), \mathrm{n}=8$. (F), Phosphorylation of ErbB4 after spinal administration of $\mathrm{NRGI}(\mathrm{I} 0 \mu \mathrm{M} / 5 \mu \mathrm{L})$, $\mathrm{n}=6$. ( $\mathbf{G}$ and $\mathbf{H})$, Effect of NRGI $(I 0 \mu M / 5 \mu L)$ on the mechanical allodynia $(\mathbf{G})$ and thermal hyperalgesia $(\mathbf{H})$ in naive mice, $n=8$. (I and J), Effect of ErbB4 siRNA on the NRGIinduced mechanical allodynia $(\mathbf{I})$ and thermal hyperalgesia $(\mathbf{J})$ in naïve mice, $\mathrm{n}=8$.

Notes: $* P<0.05, * * P<0.01, * * * P<0.001 ;{ }^{\#} P<0.05,{ }^{\#} P<0.01$, ${ }^{\# \#} P<0.001$.

Abbreviations: ErbB4, Erb-B2 receptor tyrosine kinase 4; NRGI, neuregulin I; siRNA, small interfering RNA; CCI, chronic constriction injury.

are as follows: (1) CCI induced a significant increase in the activation, but not expression, of ErbB4 in spinal PV interneurons. (2) Knockdown of spinal ErbB4 prevented and reversed mechanical allodynia in CCI mice. In contrast, activation of spinal ErbB4 induced mechanical allodynia in naïve mice. (3) Glycine-mediated, but not GABA-mediated, disinhibition is involved in the regulation of mechanical hypersensitivity by ErbB4. These 

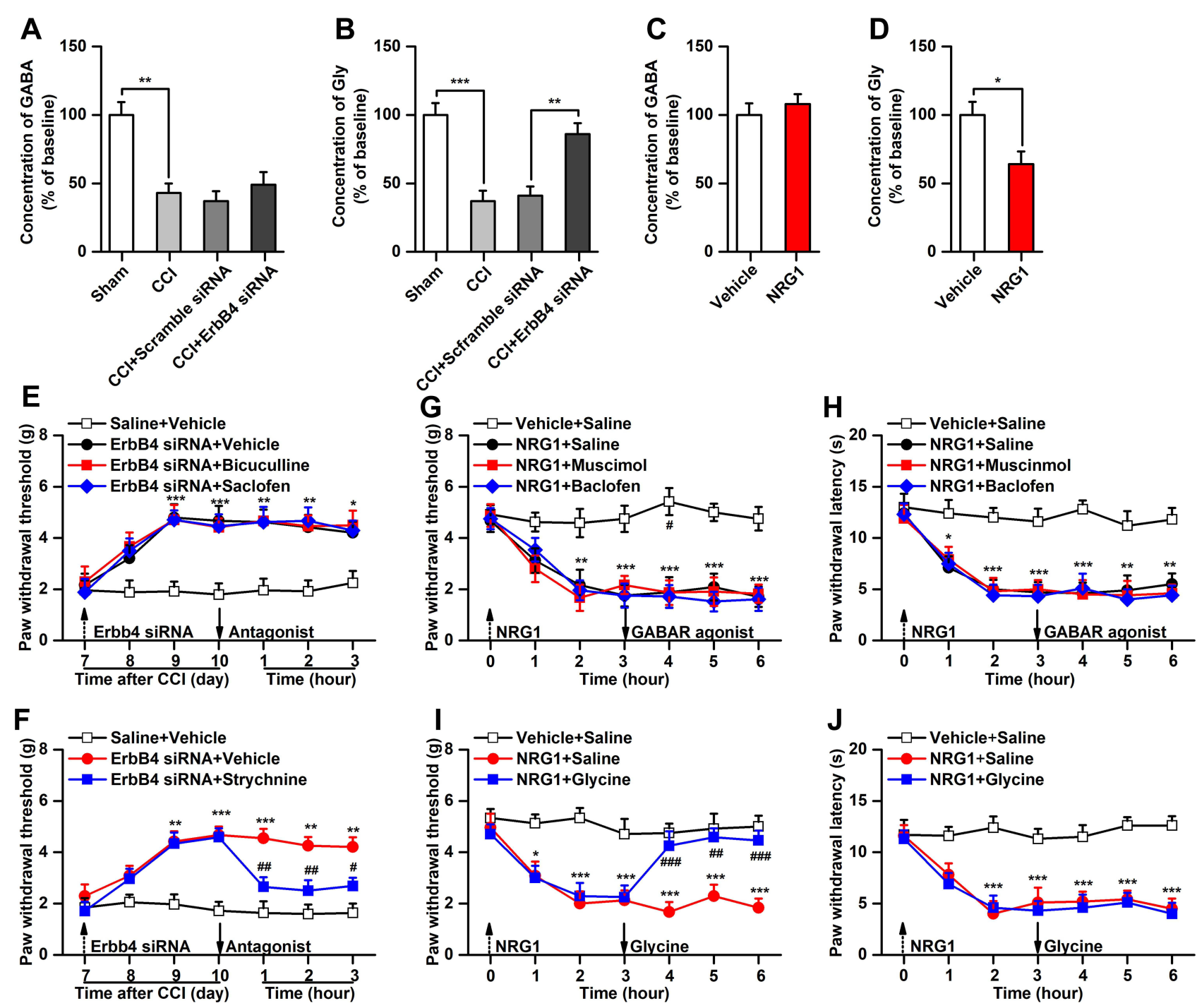

Figure 4 Inhibition of glycine release is required for the modulation of mechanical allodynia by ErbB4. (A and B), Effect of ErbB4 siRNA (I0 $\mu g / 5 \mu L)$ on the release of spinal GABA and glycine in $\mathrm{CCl}$ mice, $n=6$. (C and $\mathbf{D})$, Effect of NRGI $(10 \mu \mathrm{M} / 5 \mu \mathrm{L})$ on the release of spinal GABA and glycine in naive mice, $n=6$. (E), Bicuculline $(0.05 \mu g / 5 \mu \mathrm{L})$ and saclofen $(5 \mu \mathrm{g} / 5 \mu \mathrm{L})$ have no effect on the decreased mechanical allodynia induced by ErbB4 siRNA in $\mathrm{CCl}$ mice, $\mathrm{n}=8$. $(\mathbf{F})$, Strychnine $(\mathrm{I} \mu \mathrm{g} / 5 \mu \mathrm{L})$ reversed the decreased mechanical allodynia induced by ErbB4 siRNA in CCl mice, $n=8$. (G and $\mathbf{H})$, Glycine $(0.5 \mu \mathrm{g} / 5 \mu \mathrm{L})$, not muscimol $(0.1 \mu \mathrm{g} / 5 \mu \mathrm{L})$ or baclofen $(0.5 \mu \mathrm{g} / 5 \mu \mathrm{L})$, reversed the $\mathrm{NRGI}$ induced mechanical allodynia in naïve mice, $\mathrm{n}=8$. (I and J), Muscimol $(0.1 \mu \mathrm{g} / 5 \mu \mathrm{L})$, baclofen $(0.5 \mu \mathrm{g} / 5 \mu \mathrm{L})$, or $\mathrm{Glycine}(0.5 \mu \mathrm{g} / 5 \mu \mathrm{L})$ have no effect on the mechanical allodynia and thermal hyperalgesia induced by NRGI, $\mathrm{n}=8$.

Notes: $* P<0.05, * * P<0.01, * * * P<0.001 ;{ }^{\#} P<0.01,{ }^{\# \#} P<0.001$.

Abbreviations: ErbB4, Erb-B2 receptor tyrosine kinase 4; NRGI, neuregulin I; siRNA, small interfering RNA; CCl, chronic constriction injury.

findings demonstrated a novel mechanism for the modulation of neuropathic pain by ErbB4 in spinal PV interneurons.

Based on the gate control theory proposed by Melzack and Wall, ${ }^{1}$ under neuropathic pain conditions, mechanical allodynia may result from the dysfunction of inhibitory interneurons in the spinal dorsal horn. ${ }^{28,29}$ Recent evidence has shown that several types of inhibitory interneurons in the spinal cord play an important role in the production and persistence of mechanical hypersensitivity after nerve injury. For example, $\mathrm{Lu}$ et al reported that the inhibitory effect of lamina III glycinergic neurons on $\mathrm{PKC} \gamma$ neurons is reduced following peripheral nerve injury, leading to the amplification of pain messages that produce mechanical allodynia. ${ }^{30}$ By using intersectional genetic manipulation methods, Duan et al identified dynorphin (Dyn)-expressing inhibitory interneurons in lamina III that are necessary to gate $A \beta$ fibers from activating somatostatin $(\mathrm{SOM})+$ neurons to evoke mechanical hypersensitivity. ${ }^{31}$ Moreover, a recent study reported that dorsal horn PV interneurons in laminae IIi and III act as gatekeepers and prevent touch inputs from activating pain 
circuits. ${ }^{12}$ In the present study, by using a CCI-induced neuropathic pain model, we found for the first time that the activation of spinal ErbB4 in PV interneurons is critical for mechanical allodynia induced by nerve injury. Our findings may provide a novel drug target for mechanical allodynia prevention and treatment.

NRG1/ErbB4 signaling has been implicated in neural development, including circuitry generation, axon ensheathment, neurotransmission, and synaptic plasticity. ${ }^{14}$ In the present study, we found that activation of ErbB4 in spinal PV interneurons is important for the development and maintenance of mechanical hypersensitivity in neuropathic pain. The expression of p-ErbB4, but not ErbB4, was upregulated in PV interneurons after CCI. Knockdown of spinal ErbB4 prevented and reversed CCI-induced mechanical allodynia but not thermal hypersensitivity. However, interestingly, activation of ErbB4 by intrathecal injection of NRG1 induced both mechanical allodynia and thermal hypersensitivity in naïve mice. It has been reported that NRG1 promotes microglial proliferation and chemotaxis via the ErbB2 receptor. ${ }^{26}$ Furthermore, various studies have demonstrated that increased microgliosis could enhance pain processing. ${ }^{32}$ Concerning the role of NRG1 in microgliosis and microgliosis in pain modulation, we predict that NRG1-induced thermal hypersensitivity may occur through a microgliosisdependent mechanism. Collectively, our findings demonstrate that ErbB4 expressed in spinal PV interneurons contributes to the modulation of mechanical allodynia.

Disinhibition of spinal PV interneurons has been proposed to be essential for the production of mechanical allodynia. ${ }^{12}$ Previous studies have demonstrated that PV interneurons in the spinal dorsal horn express both GABA and glycine. ${ }^{11}$ In the present study, we found that glycine, not GABA, contributes to the ErbB4-mediated regulation of mechanical allodynia. Loss of glycinergic inhibitory tone of PV interneurons leads to reduced inhibitory control of PKC $\gamma$ interneurons, thus amplifying the transmission of pain messages that produce mechanical hypersensitivity. ${ }^{12}$ Consistently, a recent study found that increasing cerebrospinal fluid glycine content by applying glycine transporter inhibitors results in an obvious antiallodynic effect in a rat model of neuropathic pain, indicating an important role for glycine in the regulation of mechanical allodynia. ${ }^{33,34}$ Activation of ErbB4 by NRG1 has been shown to increase AMPAR endocytosis in hippocampal neurons. ${ }^{20}$ Furthermore, previous studies have indicated that AMPAR endocytosis-mediated long-term depression is involved in spinal glycinergic neuron-mediated disinhibition. ${ }^{30}$
Therefore, we predict that ErbB4 may mediate the modulation of PV interneuron-mediated disinhibition through an AMPAR endocytosis-dependent mechanism.

\section{Conclusions}

In conclusion, this study identified an NRG1 receptor, ErbB4, as an important gatekeeper involved in the development and persistence of mechanical allodynia. Our findings may lead to the discovery of novel targeted drugs for the treatment and alleviation of mechanical allodynia in neuropathic pain.

\section{Author Contributions}

All authors made a significant contribution to the work reported, whether that is in the conception, study design, execution, acquisition of data, analysis and interpretation, or in all these areas; took part in drafting, revising or critically reviewing the article; gave final approval of the version to be published; have agreed on the journal to which the article has been submitted; and agree to be accountable for all aspects of the work.

\section{Disclosure}

The authors declare no conflicts of interest in this work.

\section{References}

1. Melzack R, Wall PD. Pain mechanisms: a new theory. Science. 1965;150(3699):971-979. doi:10.1126/science.150.3699.971

2. Sandkuhler J. Models and mechanisms of hyperalgesia and allodynia. Physiol Rev. 2009;89(2):707-758. doi:10.1152/physrev.00025.2008

3. Todd AJ. Neuronal circuitry for pain processing in the dorsal horn. Nat Rev Neurosci. 2010;11(12):823-836. doi:10.1038/nrn2947

4. Zeilhofer HU, Wildner H, Yevenes GE. Fast synaptic inhibition in spinal sensory processing and pain control. Physiol Rev. 2012;92 (1):193-235. doi:10.1152/physrev.00043.2010

5. Lewis DA, Curley AA, Glausier JR, Volk DW. Cortical parvalbumin interneurons and cognitive dysfunction in schizophrenia. Trends Neurosci. 2012;35(1):57-67. doi:10.1016/j.tins.2011.10.004

6. Li KX, Lu YM, Xu ZH, et al. Neuregulin 1 regulates excitability of fast-spiking neurons through Kv1.1 and acts in epilepsy. Nat Neurosci. 2011;15(2):267-273. doi:10.1038/nn.3006

7. Tan GH, Liu YY, Hu XL, Yin DM, Mei L, Xiong ZQ. Neuregulin 1 represses limbic epileptogenesis through ErbB4 in parvalbumin-expressing interneurons. Nat Neurosci. 2011;15 (2):258-266. doi:10.1038/nn.3005

8. Verret L, Mann EO, Hang GB, et al. Inhibitory interneuron deficit links altered network activity and cognitive dysfunction in Alzheimer model. Cell. 2012;149(3):708-721. doi:10.1016/j.cell.2012.02.046

9. Yamamoto T, Carr PA, Baimbridge KG, Nagy JI. Parvalbumin- and calbindin D28k-immunoreactive neurons in the superficial layers of the spinal cord dorsal horn of rat. Brain Res Bull. 1989;23 (6):493-508. doi:10.1016/0361-9230(89)90195-0

10. Yoshida S, Senba E, Kubota Y, et al. Calcium-binding proteins calbindin and parvalbumin in the superficial dorsal horn of the rat spinal cord. Neuroscience. 1990;37(3):839-848. doi:10.1016/03064522(90)90113-I 
11. Laing I, Todd AJ, Heizmann CW, Schmidt HH. Subpopulations of GABAergic neurons in laminae I-III of rat spinal dorsal horn defined by coexistence with classical transmitters, peptides, nitric oxide synthase or parvalbumin. Neuroscience. 1994;61(1):123-132. doi:10.1016/0306-4522(94)90065-5

12. Petitjean H, Pawlowski SA, Fraine SL, et al. Dorsal Horn parvalbumin neurons are gate-keepers of touch-evoked pain after nerve injury. Cell Rep. 2015;13(6):1246-1257. doi:10.1016/j.celrep.2015.09.080

13. Mei L, Xiong WC. Neuregulin 1 in neural development, synaptic plasticity and schizophrenia. Nat Rev Neurosci. 2008;9(6):437-452. doi:10.1038/nrn2392

14. Mei L, Nave KA. Neuregulin-ERBB signaling in the nervous system and neuropsychiatric diseases. Neuron. 2014;83(1):27-49. doi:10.1016/j.neuron.2014.06.007

15. Lai C, Lemke G. An extended family of protein-tyrosine kinase genes differentially expressed in the vertebrate nervous system. Neuron. 1991;6(5):691-704. doi:10.1016/0896-6273(91)90167-X

16. Fazzari P, Paternain AV, Valiente M, et al. Control of cortical GABA circuitry development by $\mathrm{Nrg} 1$ and ErbB4 signalling. Nature. 2010;464(7293):1376-1380. doi:10.1038/nature08928

17. Vullhorst D, Neddens J, Karavanova I, et al. Selective expression of ErbB4 in interneurons, but not pyramidal cells, of the rodent hippocampus. J Neurosci. 2009;29(39):12255-12264. doi:10.1523/ JNEUROSCI.2454-09.2009

18. Wen L, Lu YS, Zhu XH, et al. Neuregulin 1 regulates pyramidal neuron activity via ErbB4 in parvalbumin-positive interneurons. Proc Natl Acad Sci U S A. 2010;107(3):1211-1216. doi:10.1073/ pnas.0910302107

19. Yau HJ, Wang HF, Lai C, Liu FC. Neural development of the neuregulin receptor ErbB4 in the cerebral cortex and the hippocampus: preferential expression by interneurons tangentially migrating from the ganglionic eminences. Cereb Cortex. 2003;13(3):252-264. doi:10.1093/cercor/13.3.252

20. Kwon OB, Longart M, Vullhorst D, Hoffman DA, Buonanno A. Neuregulin-1 reverses long-term potentiation at CA1 hippocampal synapses. $\quad J \quad$ Neurosci. 2005;25(41):9378-9383. doi:10.1523/ JNEUROSCI.2100-05.2005

21. Bennett GJ, Xie YK. A peripheral mononeuropathy in rat that produces disorders of pain sensation like those seen in man. Pain. 1988;33(1):87-107. doi:10.1016/0304-3959(88)90209-6

22. Kim SH, Chung JM. An experimental model for peripheral neuropathy produced by segmental spinal nerve ligation in the rat. Pain. 1992;50(3):355-363. doi:10.1016/0304-3959(92)90041-9
23. Richner M, Bjerrum OJ, Nykjaer A, Vaegter CB. The spared nerve injury (SNI) model of induced mechanical allodynia in mice. $J$ Vis Exp. 2011;54. doi: $10.3791 / 3092$

24. Zhou XL, Wang Y, Zhang CJ, Yu LN, Cao JL, Yan M. PKA is required for the modulation of spinal nociceptive information related to ephrinB-EphB signaling in mice. Neuroscience. 2015;284:546-554. doi:10.1016/j.neuroscience.2014.10.025

25. Chaplan SR, Bach FW, Pogrel JW, Chung JM, Yaksh TL. Quantitative assessment of tactile allodynia in the rat paw. J Neurosci Methods. 1994;53(1):55-63. doi:10.1016/0165-0270(94) 90144-9

26. Calvo M, Zhu N, Tsantoulas $\mathrm{C}$, et al. Neuregulin-ErbB signaling promotes microglial proliferation and chemotaxis contributing to microgliosis and pain after peripheral nerve injury. $J$ Neurosci. 2010;30(15):5437-5450. doi:10.1523/JNEUROSCI.5169-09.2010

27. Woo RS, Li XM, Tao Y, et al. Neuregulin-1 enhances depolarization-induced GABA release. Neuron. 2007;54 (4):599-610. doi:10.1016/j.neuron.2007.04.009

28. Baba H, Ji RR, Kohno T, et al. Removal of GABAergic inhibition facilitates polysynaptic A fiber-mediated excitatory transmission to the superficial spinal dorsal horn. Mol Cell Neurosci. 2003;24 (3):818-830. doi:10.1016/S1044-7431(03)00236-7

29. Miraucourt LS, Peirs C, Dallel R, Voisin DL. Glycine inhibitory dysfunction turns touch into pain through astrocyte-derived D-serine. Pain. 2011;152(6):1340-1348. doi:10.1016/j. pain.2011.02.021

30. Lu Y, Dong H, Gao Y, et al. A feed-forward spinal cord glycinergic neural circuit gates mechanical allodynia. J Clin Invest. 2013;123 (9):4050-4062. doi:10.1172/JCI70026

31. Duan B, Cheng L, Bourane S, et al. Identification of spinal circuits transmitting and gating mechanical pain. Cell. 2014;159 (6):1417-1432. doi:10.1016/j.cell.2014.11.003

32. Scholz J, Woolf CJ. The neuropathic pain triad: neurons, immune cells and glia. Nat Neurosci. 2007;10(11):1361-1368. doi:10.1038/ nn1992

33. Al-Khrasani M, Mohammadzadeh A, Balogh M, et al. Glycine transporter inhibitors: a new avenue for managing neuropathic pain. Brain Res Bull. 2019;152:143-158. doi:10.1016/j.brainresbull.2019.07.008

34. Mohammadzadeh A, Lakatos PP, Balogh M, et al. Pharmacological evidence on augmented antiallodynia following systemic co-treatment with GlyT-1 and GlyT-2 inhibitors in rat neuropathic pain model. Int $J$ Mol Sci. 2021;22(5):2479. doi:10.3390/ ijms 22052479
Journal of Pain Research

\section{Publish your work in this journal}

The Journal of Pain Research is an international, peer reviewed, open access, online journal that welcomes laboratory and clinical findings in the fields of pain research and the prevention and management of pain. Original research, reviews, symposium reports, hypothesis formation and commentaries are all considered for publication. The manuscript

Submit your manuscript here: https://www.dovepress.com/journal-of-pain-research-journa management system is completely online and includes a very quick and fair peer-review system, which is all easy to use. Visit http:// www.dovepress.com/testimonials.php to read real quotes from published authors. 